\title{
Anagrafare e anagrafatura
}

\author{
Luisa di Valvasone
}

PUBBLICATO: 30 SETTEMBRE 2019

S ono giunte alla redazione alcune segnalazioni riguardo all'uso crescente di due termini avvertiti dai nostri lettori come neologismi: il sostantivo anagrafatura e il verbo anagrafare, da cui il participio con valore aggettivale anagrafato. Alla base di entrambi c'e il sostantivo anagrafe (dal greco moderno anagraphé 'iscrizione, registro' composto di aná 'su' e graphée 'scritto, scrittura') per il quale il DELI indica come data di prima attestazione il I764, con un precedente molto più antico nel I4I4, a Venezia, nella forma plurale lanagrafi. Il GRADIT marca anagrafe come termine tecnicospecialistico della burocrazia col significato di "registro che riporta il numero e lo stato civile (nascite, decessi, matrimoni) degli abitanti residenti in un comune" ma anche, per estensione, "l'ufficio comunale che custodisce tale registro e rilascia i certificati a esso relativi". La formazione di anagrafare e anagrafatura a partire dal sostantivo anagrafe non stupisce: il linguaggio burocratico, a cui appartengono i nostri termini, è da sempre fortemente soggetto alla creazione di nuove parole e tra queste sono molto frequenti i verbi denominali (derivati da sostantivi) in-are e-izzare (ad esempio, disdettare da disdetta, ospedalizzare da ospedale, ecc.). Inoltre -ura è suffisso produttivo in italiano, per formare nomi da verbi e, come si legge in Serianni I989, «già in latino serviva a ricavare deverbali dal participio passato (scrīptum $\rightarrow$ scriptūra)» come ad esempio tirato (tirare) $\rightarrow$ tiratura, cotto (cuocere) $\rightarrow$ cottura. Si può dunque presumere (e le attestazioni che vedremo in seguito sosterrebbero questa ipotesi) che la lingua burocratica abbia prodotto da anagrafe il verbo denominale anagrafare (col participio anagrafato) che a sua volta avrebbe indotto alla formazione del deverbale anagrafatura.

Oltre ad anagrafe, troviamo sui dizionari anagrafico e talvolta l'avverbio anagraficamente (GRADIT e Sabatini-Coletti 2008), ma né la lessicografia passata, ottocentesca e novecentesca, né quella contemporanea registrano anagrafare e anagrafatura. Tuttavia in rete non mancano attestazioni sia del verbo sia del sostantivo, già a partire dal XIX secolo.

Per quanto riguarda anagrafare, i risultati che troviamo tra le pagine in italiano di Google (il I8/6/20I9) - anche considerando forme flesse come anagraferemo, anagrafiamo, anagrafano, anagrafava, anagrafavo, anagrafando - sono poco più di 6.ooo. Si aggirano intorno alla stessa cifra le occorrenze su Google del participio passato prevalentemente usato con valore aggettivale: 2.460 risultati per anagrafati, 2.260 per anagrafato, 8I I per anagrafata, 30 per anagrafate. Su Google Libri invece sono 98 le occorrenze della forma all'infinito del verbo (rare le forme flesse) di cui la maggior parte novecentesche e una, la prima attestazione che siamo riusciti a rintracciare e l'unica all'interno di un repertorio lessicografico, datata I863; si trova nel Dizionario di cognizioni utili specialmente alla studiosa gioventú compilato dal preside del liceo del Carmine di Torino, Nicomede Bianchi; è interessante notare come il verbo sia però definito con un sostantivo:

Anagrafare. Inscrizione appresso i Greci ne pubblici registri del nome di uno straniero a cui concedevasi la cittadinanza. Per questo gli scrittori di statistica danno il nome di Anagrafi alle tabelle della popolazione di un paese.

Non mancano, anche se decisamente contenute, attestazioni nel linguaggio giornalistico. Se nell'archivio della "Repubblica" troviamo sporadiche occorrenze del verbo, declinato anche al participio passato, solo a partire dai primi anni del Duemila, nell'archivio del "Corriere della Sera" 
troviamo alcune attestazioni novecentesche (si noti nel secondo esempio l'uso delle virgolette, probabile segnale di una percezione del termine da parte dell'autore come quantomeno non comune):

Si sono compilate delle schede le quali, distribuite agli iman e ai muktar delle contrade, si sono andate riempiendo di tutti i dati necessari a computare, specificare, qualificare, anagrafare insomma, le famiglie e gli individui della popolazione europeo-musulmano-ebrea. (Vita bengasina, "Corriere della Sera", $5 / 2 / 1913)$

Quello di via Strigelli 9, orologiaio, è morto il I5 ottobre 1980. L’Ambrogio di viale Rimembranze di Greco 40/a è pure morto, ventun anni fa, e l'intestazione del telefono è un omaggio della vedova alla sua memoria. Degli altri tre, due rientrano nel gruppetto dei quattro "anagrafati", il terzo riserba una sorpresa. (Luciano Visintin, Ambrogio Brambilla non abita piu a Milano, "Corriere della Sera", 6/12/1983)

Infine si trova anche un'attestazione nel corpus DIACORIS - corpus d'italiano che raccoglie differenti tipologie di testi a partire dal I86I fino al 200I-, in un articolo dell'"Espresso" del I974 (si noti già ora l'uso esteso di anagrafare su cui ritorneremo più avanti):

Il grande compositore è già davanti a noi, gli manca soltanto una materia teatrale tale da permettergli d i anagrafare le sue invenzioni con nomi e cognomi. (Fedele D'Amico, Puccini affoga tra i tutu, "L'Espresso", 1974)

Il sostantivo anagrafatura invece non ha attestazioni né sull'archivio della "Repubblica" né in quello del "Corriere della Sera", ma registra 3.780 risultati tra le pagine in italiano di Google (il I8/6/20rg) e una decina su Google Libri, questi ultimi esclusivamente in scritti novecenteschi, il primo dei quali datato I935:

Ai proprietari può essere consentito che la marchiatura ed anagrafatura del loro bestiame sia fatta in locale diverso e distante da quello ordinario. (La legislazione italiana, A. Giuffrè, 1935)

Se guardiamo ai contesti, possiamo facilmente verificare che sia il verbo sia il sostantivo rimangono legati all'ambito burocratico, lo stesso ambito della parola da cui derivano, anagrafe, e della quale si portano dietro il significato specialistico di 'inserire determinati dati all'interno di un registro anagrafico' (anagrafare) e 'la procedura stessa di registrazione' (anagrafatura). Tuttavia, le attestazioni trovate in rete suggeriscono al contempo lo spostamento verso un uso più esteso che si discosta dall'accezione prettamente burocratica e si allarga verso un generico significato di 'registrare, catalogare' (e 'registrazione, catalogazione'). I due termini sono ben attestati nell'ambito aziendale e amministrativo con espressioni del tipo: anagrafare $i$ dipendenti, anagrafare $i$ rifiuti, anagrafatura degli articoli di un magazzino, anagrafatura di bombole per il gas, ecc.:

Nel protocollo, infatti, la Regione Autonoma della Sardegna si è impegnata tra le altre cose [...] ad intensificare il controllo sul territorio da parte degli organi di vigilanza al fine dell'emersione del sommerso e a completare entro il zo luglio 20 I2 l'anagrafatura di tutto il patrimonio suinicolo. (La crisi del comparto suinicolo sardo: come uscirne?, www.confagricoltura.sardegna.it, 13/5/2013)

Ci sono altri villaggi di questo tipo, anche se quello di Masseria Boncuri è il più grande, ed Emiliano ha commentato: «[...] Qui c’è un modo civile, anche dianagrafare le persone, perché per entrare bisogna dare il nome e il cognome, e non essere clandestini, avere un contratto di lavoro, e quando la polizia vuole controllare dove va una persona, sa da dove il lavoratore esce e dove arriva». (Bianca Chiaritti, Emiliano: "Centri d'accoglienza. Puglia modello per l'Italia contro il caporalato», "LaGazzettadelMezzogiorno.it", $9 / 8 / 2018)$ 
Il software Assist dedicato al settore dei centri di assistenza e riparazione consente anche la gestione delle risorse utilizzate. Infatti è possibile anagrafare sia i tecnici impiegati, che le risorse tecniche utilizzate (auto, strumentazione, laboratori, etc.). (www.progettosoftware.net)

Vi è poi un settore in cui il verbo anagrafare e il sostantivo anagraftura sembrano essersi particolarmente affermati negli ultimi anni: quello veterinario, legato comunque a procedure amministrative. La maggior parte dei contesti trovati in rete riguarda infatti l'anagrafatura di animali (di anagrafatura del bestiame si parlava già nel testo del $1935 \mathrm{e}$ in alcuni atti parlamentari del I960) e in particolare l'anagrafatura canina (55I risultati del sintagma su Google; $55^{2}$ per "anagrafatura dei cani"), ovvero l'identificazione e la registrazione di un cane, attraverso l'inserimento di un microchip sottocutaneo, all'interno della cosiddetta anagrafe canina. La procedura è oggi obbligatoria e negli ultimi venti anni lo Stato e le regioni hanno emesso diverse disposizioni che la regolassero, nelle quali è possibile ritrovare alcune occorrenze del verbo e del sostantivo:

A seguito dell'esperienza pilota di anagrafatura e considerata la validità della metodologia è previsto per tutta la Sardegna lo stesso tipo di anagrafe; ciò comporta quindi la necessità di approvvigionamento di microchip sottocutanei. (Regione Sardegna, decreto del presidente della giunta 4 marzo I999, n. I, art. I4, Criteri di anagrafatura dei cani)

Ritenuta, altresì, la necessità e l'urgenza di far effettuare in maniera contestuale l'identificazione e la registrazione di tutta la popolazione canina presente sul territorio nazionale, utilizzando strumenti e modalità uniformi per tutte le regioni e province autonome, allo scopo di anagrafare il maggior numero possibile degli animali in questione e consentirne un controllo ed una gestione adeguati. (Ministero del Lavoro, della Salute e delle Politiche Sociali, Ordinanza 6 agosto 20o8)

Frequente l'uso del participio nella forma cane/i anagrafato/i. Tra le pagine in italiano di Google (il I8/7/2019) la sequenza "cani+anagrafati" compare 2.200 volte, "anagrafare+cani" 2.990 volte e "anagrafatura+cani" I. 530 volte:

Questa Amministrazione promuove una campagna di anagrafatura canina da realizzarsi con la microchipatura gratuita. [...] Chiunque possieda un cane non ancora anagrafato, potrà presentarsi, munito di documento di identità e codice fiscale, col suo amico a quattro zampe, presso il Palazzo Comunale, piano terra, in Piazza Repubblica il giorno I8.03.20I4 dalle ore 9,00 alle ore I3,oo e dalle ore I6 alle ore I9,00 dopo essersi prenotato. (avviso pubblicato sul sito del Comune di Assemini, I4/3/2014)

Tutti i rzo cani randagi dell'isola sono ora sterilizzati, anagrafati e microchippati a nome del Sindaco. (Randagismo: siamo di nuovo sul campo per i cani e i gatti di Lampedusa!, www.lav.it, 3/6/2019)

L'affermarsi di anagrafare e anagrafatura nella regolamentazione dell'anagrafe canina sembra risalire ai primi anni del Duemila, anno della prima attestazione nell'archivio della "Repubblica" (e subito successivo alle disposizioni della Regione Sardegna del 1999):

Sui 38 pit bull confiscati alla camorra, ne restano ro adottabili, rieducati, sterilizzati e anagrafati. (Allevamento abusivo. Sequestrati pitbull a Secondigliano, "LaRepubblica.it", 8/7/200o)

Tuttavia, da una rapida indagine tra gli addetti ai lavori (per la quale si ringrazia Valentina Berti) sembra che l'uso non sia ancora totalmente penetrato nel lessico specialistico: su i63 risposte ricevute pubblicando un post in un noto gruppo su Facebook di veterinari italiani, soltanto una decina (provenienti da diverse regioni) ha affermato di usare il verbo nel suo lavoro e pochi altri di averlo sentito usare dai colleghi; da alcune testimonianze si evince che è possibile, anche se raro, imbattersi in espressioni come il cane deve essere anagrafato al posto della forma più comune il cane deve essere 
iscritto all'anagrafe.

In ogni caso, l'uso di anagrafare e anagrafatura nella veterinaria, in particolare quella riguardante gli animali domestici come i cani (presenti nella vita di molti parlanti), potrebbe aver agevolato l'estensione di significato e d'uso. A partire dall'anagrafatura canina l'argomento è diventato di dominio più pubblico e meno amministrativo, incrementando gli usi estesi dianagrafare e anagrafatura anche al di fuori dell'ambito burocratico. Come si è visto dagli esempi, anagrafare e anagrafatura si trovano oggi in contesti allargati, con un cambio evidente per quanto riguarda l'oggetto anagrafato e la funzione, non necessariamente di tipo amministrativo o legata a un obbligo rispetto a un ente pubblico, fino ad arrivare ad usi estesi come nel seguente esempio in cui il verbo sembra assumere un generico senso di 'inventariare':

Nel frattempo erano arrivate delle novità, e il libraio apriva le scatole, anagrafando i titoli nuovi, valutandoli, decidendo la sistemazione giusta. (Alberto Schiavone, La libreria dell'armadillo, Bologna, Rizzoli, 20I2)

Tale allargamento degli usi potrebbe contribuire alla progressiva affermazione di anagrafare/anagrafatura anche nel linguaggio comune. Solo il tempo potrà dirci se i due termini entreranno a pieno titolo nei linguaggi specialistici visti finora e nell'uso comune.

\section{Cita come:}

Luisa di Valvasone, Anagrafare e anagrafatura , "Italiano digitale", 2019, X, 2019/3 (lugliosettembre)

DOI: $10.35948 / 2532-9006 / 2020.3150$

\section{Copyright 2019 Accademia della Crusca}

Pubblicato con licenza creative commons CC BY-NC-ND 